\title{
3D view synthesis with inter-view consistency
}

\author{
David Wolinski \\ david.wolinski@inria.fr
}

\author{
Olivier Le Meur \\ olivier.le_meur@irisa.fr \\ University of Rennes 1 - IRISA \\ Campus de Beaulieu \\ 35042 Rennes, France
}

\author{
Josselin Gautier \\ josselin.gautier@irisa.fr
}

\begin{abstract}
In this paper, we propose a new pipeline to synthesize virtual views. It allows us to generate virtual views far away from each other, each presenting the exact same level of quality. This inter-view consistency is key to seamlessly navigate between viewpoints. Its computational cost is also lower than that of existing approaches. We compare the proposed approach with state-of-the-art methods and show the effectiveness of this new view synthesis pipeline.
\end{abstract}

\section{Categories and Subject Descriptors}

I.4.9 [Image Processing and Computer Vision]: Applications

\section{Keywords}

DIBR, FVV, 3DTV, view synthesis

\section{INTRODUCTION}

Virtual view synthesis consists in generating a new viewpoint from existing camera viewpoints. The most popular technique to synthesize virtual views is the Depth Image Based Rendering which relies on the depth information and allows to freely navigate in a scene. This application is called Free-Viewpoint Video (FVV) where virtual views are interpolated between two known ones [6] or extrapolated from one reference view. Unfortunately, DIBR methods suffer from several problems such as the recovery of disoccluded areas. Most of the time, an inpainting method is used to fillin these areas. For instance, Daribo et Pesquet-Popescu [2] extended Criminisi's algorithm [1] by modifying the priority function and the patch matching criteria. Gautier et al. [3] also modified Criminisi's approach by taking into account a direction of filling, depending on the projection direction. Ma et al. [4] proposed a depth-guided inpainting using a new priority scheme and a background-foreground separation technique. Sun et al. [5] proposed a new framework using a joint optimization of inter-view texture similarity

Permission to make digital or hard copies of all or part of this work for personal or classroom use is granted without fee provided that copies are not made or distributed for profit or commercial advantage and that copies bear this notice and the full citation on the first page. To copy otherwise, to republish, to post on servers or to redistribute to lists, requires prior specific permission and/or a fee.

WOODSTOCK' $97 \mathrm{El}$ Paso, Texas USA

Copyright 20XX ACM X-XXXXX-XX-X/XX/XX ...\$10.00. and geometry preservation. All these approaches provide new advances in view synthesis methods. However, they all overlook an important aspect which is the consistency between virtual views.

The goal of the presented work is to improve the quality of synthesized views in a context of FVV. Unlike for Zitnick et al. [6], virtual views are here extrapolated from one reference view (one texture image and its depth map).Traditionally, this is performed in the following order: (i) view projection which leads to cracks and disocclusions, (ii) crack filling which usually uses neighboring data and, finally, (iii) inpainting which aims to fill the disocclusions. We argue that the traditional pipeline, as illustrated in figure 1 (a) suffers from four main issues which essentially cripple the inpainting algorithms.

The first issue is foreground/background differentiation where inpainting algorithms are tempted to use foreground data when filling background disocclusions, leading to very disturbing "spillings". Moreover, as objects found in the scene don't always have the same edges on the texture and depth images, after projection, some foreground pixels can end up on the background and vice-versa. This "ghosting" further complicates the inpainting algorithm's job.Heuristics have been imagined, strengthening the inpainting algorithm but the problem, though minimized, largely persists.

The second issue is data corruption, which degrades the inpainting before it even started. This is caused by the inpainting algorithm using post projection, post crack-filling data.

The third issue concerns the lack of consistency between virtual views synthesized from the same reference view (as illustrated by the quality of local regions in figure 1 (a) right). For instance, a disocclusion that is visible in several virtual views is inpainted separately in each of these views. Since the point of view is not the same, this disocclusion is inpainted with differing data, producing different results which are immediately noticeable.

The fourth, last, issue lies with performance while synthesizing several views, when as mentioned, the inpainting step is done separately for each view. This not only introduces inconsistencies but is also redundant in that a disocclusion is inpainted once for each time it appears in a virtual view.

We aim to solve these issues, regardless of the inpainting algorithm that is used by essentially reversing the classical order of operations required to synthesize virtual views (figure $1(\mathrm{~b})$ ). The overall idea is to inpaint the disoccluded areas before projection automatically removing any redundancy as each disocclusion is inpainted only once and also 


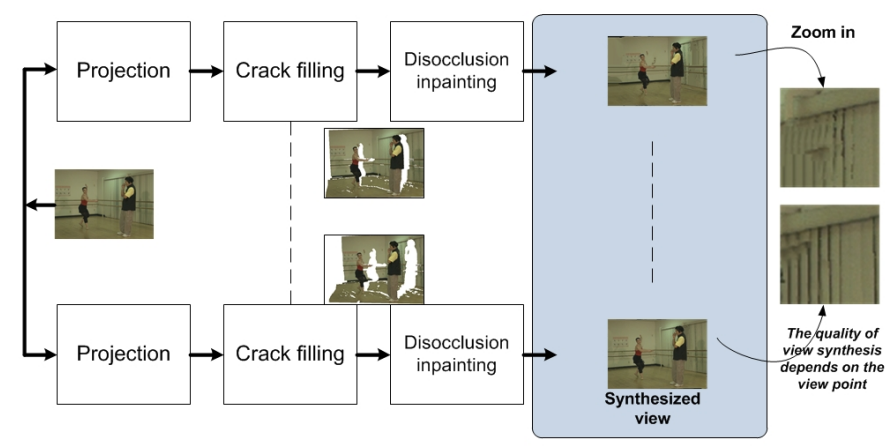

(a) Common method

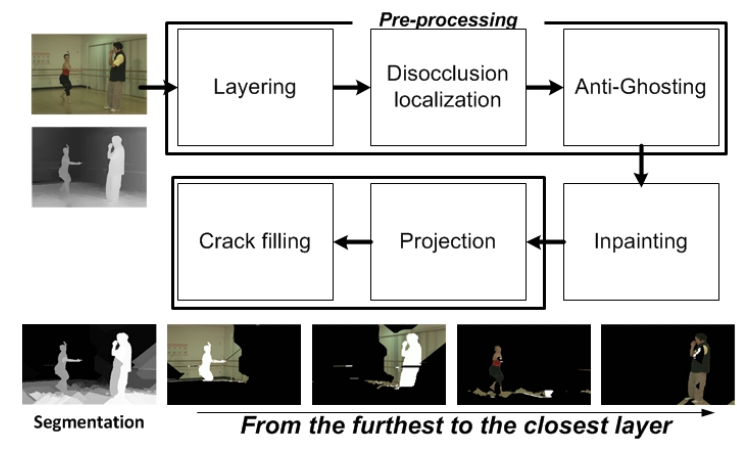

(b) Proposed method

Figure 1: (a) Common method to synthesize a virtual view. First, the original view is projected. Second, disocclusions and cracks are inpainted. (b) Proposed method to synthesize virtual views - reversed order.

guaranteeing consistency between synthesized views. The data the inpainting algorithm uses is also free of any projection errors or cracks. Finally, we locate future disocclusions on the corresponding backgrounds separate from the foregrounds, allowing anti-ghosting and avoiding "spillings".

The paper is organized as follows. In section 2 , the proposed pipeline is presented. Section 3 shows its performance and a comparison with state-of-the-art results. Conclusions are drawn in section 4

\section{PROPOSED METHOD}

The proposed approach is composed of three main sequential operations (pre-processing, inpainting and projection) described in the following sections.

\subsection{Pre-processing}

First, the pre-processing projects the reference view into the extreme views in order to detect the largest possible disocclusions. With this information, we can then separate the various foregrounds and backgrounds into layers and apply any necessary anti-ghosting.

Depth-based segmentation: The first step is a depthbased segmentation which looks for pixels that are separated by a disocclusion and regroups pixels that remain connex after projecting into extreme virtual views (furthest from the reference view). The goal being to detect disocclusions, it is appropriate to use an edge-based segmentation of the depth map. Moreover, disocclusions should only appear between layers, meaning that the edges of our segmented depth map are the borders of the disocclusions. We thus define disocclusions as a loss of connexity between two pixels $\left(p, p^{\prime}\right)$ after projection (Proj): $\forall p, p^{\prime} \in$ Image, $\left\{p, p^{\prime}\right\} \in I E P$ iff

$$
p^{\prime} \in \operatorname{neighbor} s(p) \wedge \operatorname{dist}\left(\operatorname{Proj}(p), \operatorname{Proj}\left(p^{\prime}\right)\right)>\Delta
$$

Two pixels which lose their connexity (edge-pixels) form an inter-exclusive pair $(I E P)$ : they are on each side of a future disocclusion and one of them is part of the corresponding foreground while the other is part of the corresponding background (see figure 2 (left)). Then, these edge-pixels are regrouped into regions which do not contain any interexclusive pairs (see figure 2 (middle)). All that remains to be done now is to assign all remaining pixels to one of these regions (see figure 2 (right)).

Layering: The depth-based segmentation leads to an over-segmented map. Indeed, some regions are separated by inter-exclusive pairs of pixels indicating that these regions
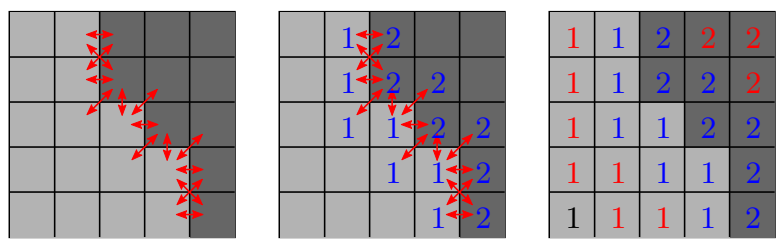

Figure 2: Left: double arrows represent interexclusive pixel pairs. Middle: labeled edge-pixels. Right: whole picture labeled, colors represent successive iterations of the region-growing algorithm.

belong to the foreground and background of a corresponding future disocclusion. However, this is not necessarily the case for all pairs of regions. The layering process aims to regroup into layers the regions that do not share any inter-exclusive pairs of pixels and that are close in terms of depth, that is, regions which do not create disocclusions amongst them. This is done by using an algorithm consisting of two nested loops as described in (Alg.1). As a result, the scene is not

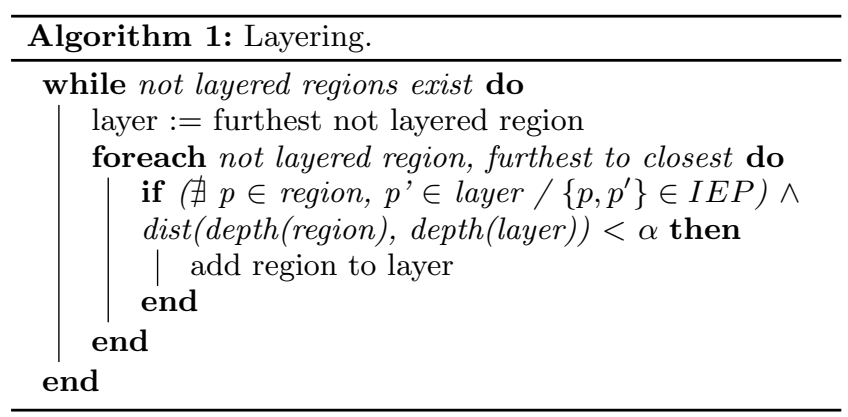

over-segmented anymore and the resulting layers are ready for the remaining steps. Figure 1 (b) (bottom) illustrates some layers and segmentation.

Disocclusion localization between layers: As ensured by the way layers are constructed, disocclusions can only appear between layers and not inside any single layer. Thanks to this property, it is possible to easily locate the future disocclusions. To do this, we once again turn to the extreme projections. For every pair of layers $L_{1}$ and $L_{2}$, if these two layers share inter-exclusive pairs of pixels, it means that they share a common future disocclusion and that the further layer, say $L_{1}$, is the background while the closer layer, 
Layer $L_{1}$

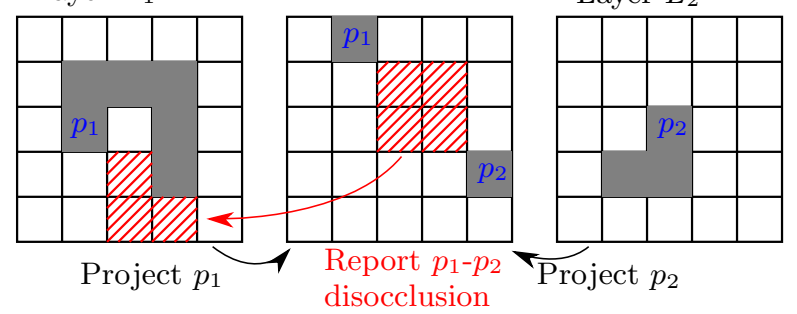

Figure 3: Left: further layer $L_{1}$. Right: closer layer $L_{2}$. Middle: pixels $p_{1}$ and $p_{2}$ projected. Red: discovered disocclusion reported on $L_{1}$.

say $L_{2}$, is the foreground. Now, considering each of these inter-exclusive pairs of pixels, let $p_{1}$ be the one belonging to layer $L_{1}$ (figure 3 (left)) and $p_{2}$ the one belonging to layer $L_{2}$ (figure 3 (right)). In the extreme projections, $p_{1}$ and $p_{2}$ lose connexity by a number of pixels greater than $\Delta$ (figure 3 (middle)). All corresponding pixels that are unknown on the $L_{1}$ layer (background layer) are then marked as disocclusion (reported pixels in red in figure 3 (left)). When this process is finished for all inter-exclusive pairs, all disocclusions have been found. More importantly, these disocclusions are now associated with the corresponding backgrounds which ensures background/foreground differentiation. This is a key point of the proposed approach and especially for the inpainting process (see section 2.2).

Anti-ghosting: The last phase of the pre-processing is the anti-ghosting which erodes known data around marked disocclusions. This way, any foreground data (according to the texture image) that can end up on the background (according to the depth-map) is erased and is not considered by the inpainting algorithm when filling the disocclusions.

\subsection{Inpainting}

The pre-processing allows us to decompose the scene into layers in which the disocclusions have been detected. Next, the disocclusions are inpainted using the algorithm (Alg.2).

In our case, we first inpaint the depth values by interpolating them and then inpaint the texture values using the algorithm presented in [3]. Note that we could have chosen another inpainting algorithm or improved it. However, this point is out of the scope of this paper.

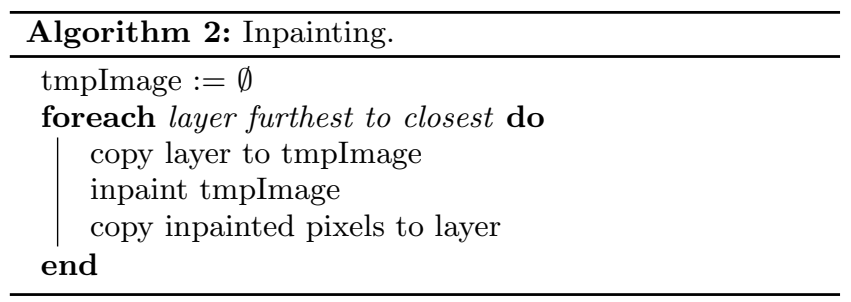

\subsection{Projection and crack-filling}

The last thing that remains to be done is to effectively synthesize the virtual views. The algorithm is shown in (Alg.3), where a view is assembled in "viewImage". Importantly, views are synthesized fast, without additional inpainting.

\section{RESULTS}
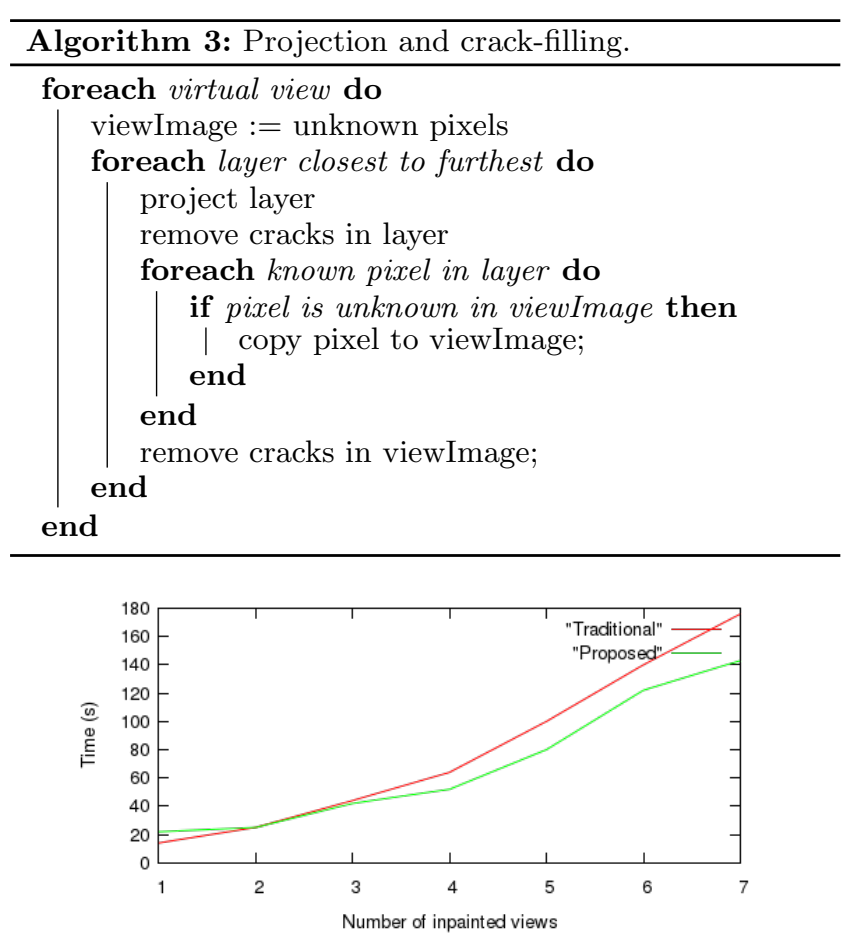

Figure 4: Execution times for traditional and proposed pipelines (for same settings).

To evaluate the performance of the proposed approach, two multiview sequences, Ballet and Breakdancers, have been used [6]. They include 8 views (resolution is $1024 \times 768$ ) where the reference views are the views 5 and 3 respectively. The goal is to project these reference views into other camera viewpoints and to check if the visual quality between synthesized views is similar. We compare results with two state-of-the-art methods, Daribo [2] and Gautier [3]. As these two methods are based on depth maps, the used depth maps are the projected interpolated ones, as in the proposed approach. Figure 5 illustrates a comparison where the reference view is projected into 4 different viewpoints (we call $V_{i \rightarrow j}$, the projection of view $i$ into $j$ ). The quality between synthesized views varies a lot for Daribo and Gautier's methods, whereas with the proposed approach, the quality is exactly the same ensuring a seamless transition between synthesized views. In other words, when a user wants to move his viewpoint, the quality level stays perfectly consistent. Also note that on Ballet, only the proposed approach correctly fills the hole between the man's arm and chest and correctly differentiates the woman from the wall. Figure 4 gives the running time of the proposed approach and a traditional one [3] in function of the number of synthesized views. When this number increases, the proposed approach clearly outperforms method [3]. Figure 6 illustrates the results of the proposed approach on the sequence BreakDancers. The quality is, again, the same on the different projected views. Note that a quantitative assessment is not provided due to both the ill-posed nature of the inpainting approach and the lack of space. More results on http://people.irisa.fr/Olivier.Le_Meur/.

\section{CONCLUSION}

We present a new pipeline to synthesize virtual views. In order to ensure inter-view consistency (in terms of quality), 


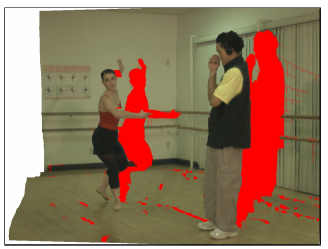

(a) $V_{5 \rightarrow 3}$

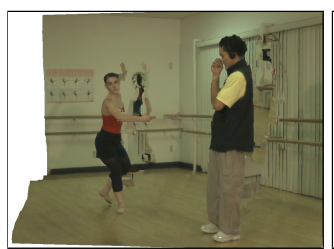

(f) $V_{5 \rightarrow 3}[2]$

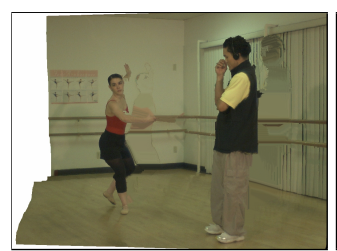

(k) $V_{5 \rightarrow 3}[3]$

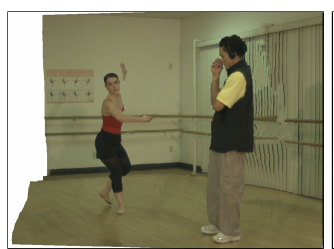

(p) Proposed $V_{5 \rightarrow 3}$

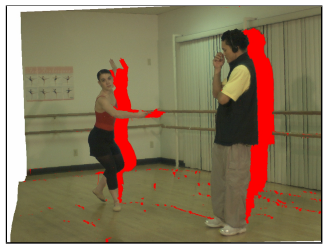

(b) $V_{5 \rightarrow 4}$

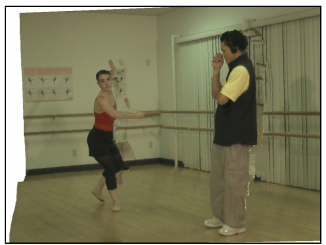

(g) $V_{5 \rightarrow 4}[2]$

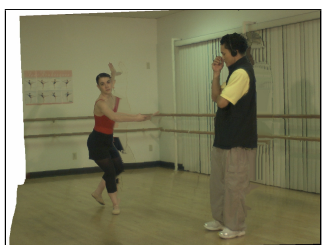

(l) $V_{5 \rightarrow 4}[3]$

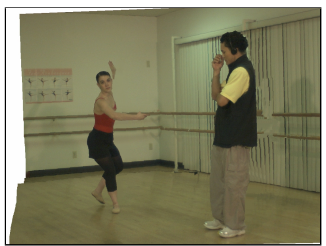

(q) Proposed $V_{5 \rightarrow 4}$

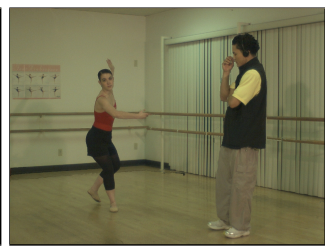

(c) Reference view $V_{5}$

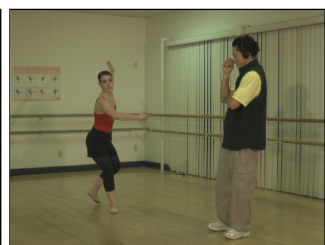

(h) Reference view $V_{5}$

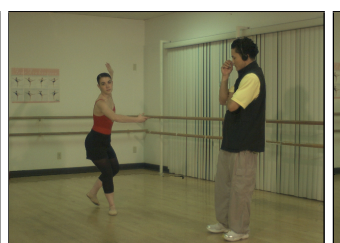

(m) Reference view $V_{5}$

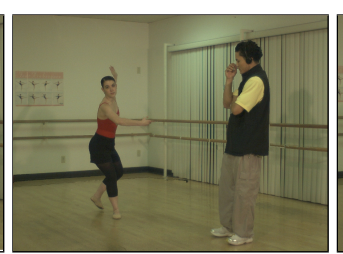

(r) Reference view $V_{5}$

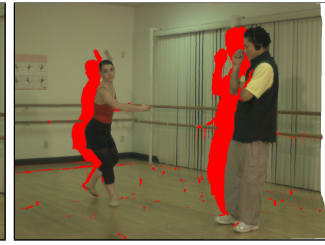

(d) $V_{5 \rightarrow 6}$

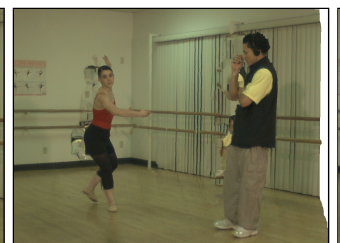

(i) $V_{5 \rightarrow 6}[2]$

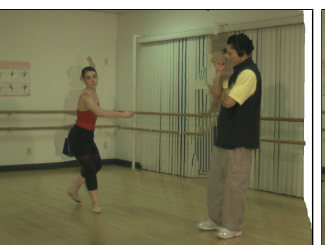

(n) $V_{5 \rightarrow 6}[3]$

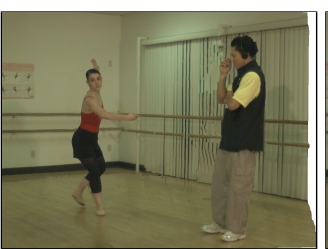

(s) Proposed $V_{5 \rightarrow 6}$

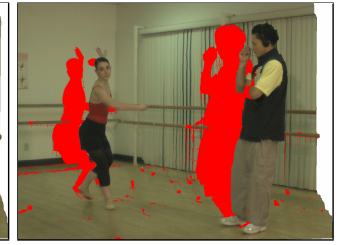

(e) $V_{5 \rightarrow 7}$

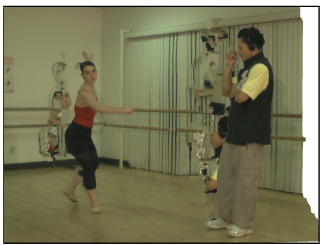

(j) $V_{5 \rightarrow 7}[2]$

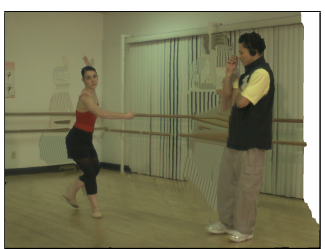

(o) $V_{5 \rightarrow 7}[3]$

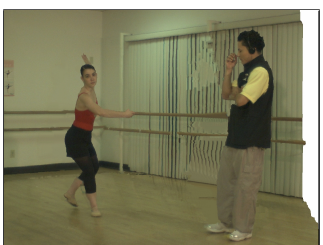

(t) Proposed $V_{5 \rightarrow 7}$

Figure 5: Virtual view synthesis on the sequence Ballet. (a) to (e): the view $V_{5}$ is projected into 4 viewpoints. Disoccluded areas are in red. (f) to (j): inpainting results of [2]; (k) to (o): inpainting results of [3]; (p) to $(t)$ : proposed approach. Note the quality of our results is superior and the same whatever the projection.

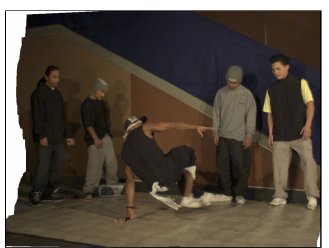

(a) Proposed $V_{3 \rightarrow 1}$

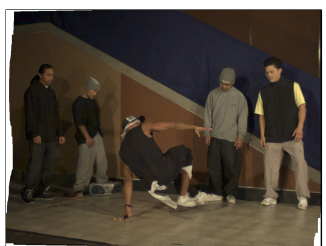

(b) Proposed $V_{3 \rightarrow 2}$

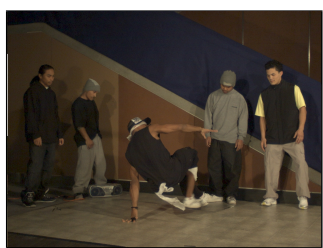

(c) Reference view $V_{3}$

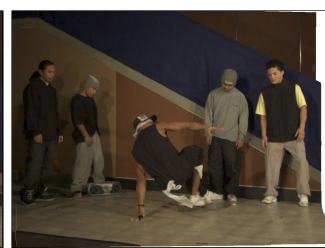

(d) Proposed $V_{3 \rightarrow 4}$

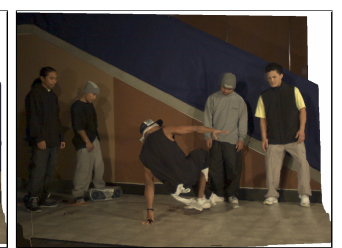

(e) Proposed $V_{3 \rightarrow 5}$

Figure 6: Virtual view synthesis on the sequence BreakDancers.

we propose to inpaint the disoccluded areas before projection. Results show the effectiveness of this approach both in terms of running time and overall quality. A future study would be to consider more than one reference view. The temporal dimension is also an interesting avenue.

\section{REFERENCES}

[1] A. Criminisi, P. Pérez, and K. Toyama. Region filling and object removal by exemplar-based image inpainting. IEEE trans. on Image Processing, 13:1200-1212, 2004.

[2] I. Daribo and B. Pesquet-Popescu. Depth-aided image inpainting for novel view synthesis. In Multimedia Signal Processing (MMSP), pages 167-170, 2010.
[3] J. Gautier, O. Le Meur, and C. Guillemot. Depth-based image completion for view synthesis. In $3 D T V$ Conference, 2011.

[4] L. Ma, L. Do, and P. de With. Depth-guided inpainting algorithm for free-viewpoint video. In ICIP, pages 1721-1724, 2012.

[5] W. Sun, C. A. Oscar, L. Xu, Y. Li, W. Hu, and Z. Yu. Extended criminisi's algorithm by modifying the priority function and the patch matching criteria. In ACM Multimedia, pages 733-736, 2012.

[6] C. Zitnick, S. Kang, M. Uyttendaele, S. Winder, and R. Szeliski. High-quality video view interpolation using a layered representation. In $A C M S I G G R A P H$, pages 600-608, 2004. 